\title{
A high-order finite difference scheme for a singularly perturbed reaction-diffusion problem with an interior layer
}

\author{
Zhongdi Cen ${ }^{1,2}$, Anbo Le ${ }^{2^{*}}$ and Aimin Xu
}

\section{"Correspondence: \\ anbole7@hotmail.com \\ ${ }^{2}$ Institute of Mathematics, Zhejiang \\ Wanli University, Ningbo, China \\ Full list of author information is \\ available at the end of the article}

\begin{abstract}
In this paper, we consider a singularly perturbed reaction-diffusion problem with a discontinuous source term. Boundary and interior layers appear in the solution. The problem is discretized by using a hybrid finite difference scheme on a Shishkin-type mesh. A nonequidistant generalization of the Numerov scheme is used on the Shishkin-type mesh except for the point of discontinuity, whereas a second-order difference scheme with an additional refined mesh is used for the point of discontinuity. Although the difference scheme does not satisfy the discrete maximum principle, the maximum norm stability of the scheme is established. The maximum error in the mesh points is shown to be uniformly bounded by $\left(N^{-1} \ln N\right)^{4}$ with a constant independent of the perturbation parameter. Numerical results supporting the theory are presented.
\end{abstract}

MSC: $65 \mathrm{~L} 10 ; 65 \mathrm{~L} 12$

Keywords: singular perturbation; reaction-diffusion equation; Shishkin-type mesh; finite difference scheme; uniform convergence

\section{Introduction}

We consider the following singularly perturbed reaction-diffusion problem with a discontinuous source term:

$$
\begin{aligned}
& L u(x) \equiv-\varepsilon^{2} u^{\prime \prime}(x)+b(x) u(x)=f(x), \quad x \in \Omega^{-} \cup \Omega^{+}, \\
& u(0)=A, \quad u(1)=B,
\end{aligned}
$$

where $0<\varepsilon \ll 1$ is the perturbation parameter, $A$ and $B$ are given constants, $b \geq \beta^{2}>0$ is a sufficiently smooth function on $\bar{\Omega}, \Omega^{-}=(0, d), \Omega^{+}=(d, 1)$, and $\Omega=(0,1)$. We assume that the function $f$ is sufficiently smooth on $\Omega^{-} \cup \Omega^{+}$and has a jump discontinuity at the point $d \in \Omega$. These hypotheses ensure that problem (1.1)-(1.2) has a unique solution $u \in C^{1}(\Omega) \cap C^{2}\left(\Omega^{-} \cup \Omega^{+}\right)$(see $\left.[1,2]\right)$. For $\varepsilon \ll 1$, the solution $u$ has boundary and interior layers. It is shown that such a problem arises naturally in the context of models of simple semiconductor devices [2].

Due to the presence of these layers, classical numerical methods are not appropriate to numerically solve singularly perturbed problems. Special methods are required for ob-

(c) The Author(s) 2017. This article is distributed under the terms of the Creative Commons Attribution 4.0 International License (http://creativecommons.org/licenses/by/4.0/), which permits unrestricted use, distribution, and reproduction in any medium, provided you give appropriate credit to the original author(s) and the source, provide a link to the Creative Commons license, and indicate if changes were made. 
taining good numerical approximations to such problems. Singularly perturbed reactiondiffusion equations with sufficiently smooth data have been studied extensively; see, for instance, $[3,4]$ for a survey. However, only few results for singularly perturbed reactiondiffusion equations with nonsmooth data are reported in the literature. Miller et al. [2] proposed a parameter-uniform Schwarz method on a Shishkin mesh for problem (1.1)-(1.2) and proved that the scheme is first-order convergent in the discrete maximum norm. Roos and Zarin [5] introduced a Galerkin finite element method with a Bakhvalov-Shishkin mesh for problem (1.1)-(1.2) and showed that the scheme is second-order convergent in the discrete maximum norm. Chandru et al. [1] presented a second-order hybrid difference scheme on a Shishkin mesh for problem (1.1)-(1.2). Farrell et al. [6] and Boglaev and Pack [7] employed first-order uniformly convergent difference schemes for singularly perturbed semilinear differential equations with a discontinuous source term. Falco and O'Riordan [8] developed a second-order uniformly convergence numerical method on piecewise-uniform Shishkin meshes for a reaction-diffusion equation with a discontinuous diffusion coefficient. Rao and Chawla [9] used a first-order convergent difference scheme for a coupled system of singularly perturbed reaction-diffusion equations with discontinuous source terms. Brayanov [10] constructed a finite volume difference scheme for two-dimensional versions of problem (1.1)-(1.2). Since the source term $f$ is discontinuous at the point $x=d$, in general the solution $u$ of problem (1.1)-(1.2) has no continuous high-order derivatives at the point $x=d$, which leads to the numerical difficulty for constructing high-order numerical schemes.

In this paper, we propose a high-order finite difference scheme on a Shishkin-type mesh for problem (1.1)-(1.2). A nonequidistant generalization of the Numerov scheme is used on the Shishkin-type mesh except for the point of discontinuity $x=d$, whereas a second-order difference scheme with an additional refined mesh is used for $x=d$. Although the difference scheme does not satisfy the discrete maximum principle, we show that the scheme is maximum-norm stable. We prove that the scheme has accuracy $O\left(\left(N^{-1} \ln N\right)^{4}\right)$, uniformly in the perturbation parameter. Our hybrid difference scheme for problem (1.1)-(1.2) is a modification of the Numerov scheme used in [11-14] for singularly perturbed reactiondiffusion problems with sufficiently smooth data.

An outline of the paper is as follows. In the next section, we present some analytical results of the boundary value problem (1.1)-(1.2). The discrete scheme is described in Section 3. The stability and convergence properties of the numerical scheme are given in Section 4. Numerical examples are presented in support of our theoretical estimates in Section 5. Finally, the conclusion is given in Section 6.

Notation Throughout the paper, $C$ will denote a generic positive constant that is independent of $\varepsilon$ and the mesh. Note that $C$ is not necessarily the same at each occurrence. Assume that $g(x)$ is a function on a close set $D$ and $\omega$ is a discretization mesh of $D$. To simplify the notation, we denote the jump of the function $g(x)$ at $d \in D$ by $[g](d)=g(d+)-g(d-)$. We set $g_{i}=g\left(x_{i}\right)$ and let $G_{i}$ denote a numerical approximation of $g(x)$ at $x_{i} \in \omega$. We also define $\|g\|_{D}=\max _{x \in D}|g(x)|$ and $\|G\|_{\omega}=\max _{x_{i} \in \omega}\left|G_{i}\right|$.

\section{Properties of the exact solution}

For constructing layer-adapted meshes correctly, we need to know the asymptotic behavior of the exact solution. This behavior will be used later in the analysis of the uniform convergence of the finite difference scheme defined in Section 3. 
Lemma 2.1 Suppose $b \in C^{4}(\bar{\Omega})$ and $f \in C^{4}\left(\Omega^{-} \cup \Omega^{+}\right)$. Then the solution $u$ of problem (1.1)(1.2) can be decomposed as

$$
u(x)=v(x)+w(x)
$$

where the regular solution component $v(x)$ satisfies

$$
\begin{aligned}
& L v(x)=f(x), \quad x \in \Omega^{-} \cup \Omega^{+}, \\
& v(0)=f(0) / b(0), \quad v(d-)=f(d-) / b(d), \\
& v(d+)=f(d+) / b(d), \quad v(1)=f(1) / b(1),
\end{aligned}
$$

and

$$
\left|v^{(k)}(x)\right| \leq C\left(1+\varepsilon^{4-k}\right), \quad x \in \Omega^{-} \cup \Omega^{+}, 0 \leq k \leq 6,
$$

whereas the singular solution component $w(x)$ satisfies

$$
\begin{aligned}
& L w(x)=0, \quad x \in \Omega^{-} \cup \Omega^{+}, \\
& w(0)=u(0)-v(0), \quad w(1)=u(1)-v(1), \\
& {[w](d)=-[v](d), \quad\left[w^{\prime}\right](d)=-\left[v^{\prime}\right](d),}
\end{aligned}
$$

and

$$
\left|w^{(k)}(x)\right| \leq\left\{\begin{array}{ll}
C \varepsilon^{-k}\left(e^{-\beta x / \varepsilon}+e^{-\beta(d-x) / \varepsilon}\right), & x \in \Omega^{-}, \\
C \varepsilon^{-k}\left(e^{-\beta(x-d) / \varepsilon}+e^{-\beta(1-x) / \varepsilon}\right), & x \in \Omega^{+},
\end{array} \quad 0 \leq k \leq 6\right.
$$

Proof See [2], Lemma 1, for a proof with $0 \leq k \leq 4$; the argument there also works for $k=5,6$.

\section{Discretization}

We consider a high-order finite difference scheme on a Shishkin-type mesh for problem (1.1)-(1.2). Let our discretization parameter $N$ be divisible by 8 . Define the mesh transition parameters $\sigma_{1}$ and $\sigma_{2}$ as

$$
\sigma_{1}=\min \left\{\frac{d}{4}, \frac{4 \varepsilon}{\beta} \ln N\right\} \quad \text { and } \quad \sigma_{2}=\min \left\{\frac{1-d}{4}, \frac{4 \varepsilon}{\beta} \ln N\right\}
$$

Note that if $\sigma_{1}=\frac{d}{4}$ or $\sigma_{2}=\frac{1-d}{4}$, then $N^{-1}$ is exponentially small compared to $\varepsilon$, and therefore a classical analysis of the convergence can be made. So, here we only consider the most interesting case in practice, that is,

$$
\sigma_{1}=\sigma_{2}=\sigma, \quad \sigma=\frac{4 \varepsilon}{\beta} \ln N
$$


Then our Shishkin-type mesh is given as follows:

$$
x_{i}= \begin{cases}\frac{8 \sigma}{N} i, & 0 \leq i \leq \frac{N}{8}, \\ \sigma+\frac{4(d-2 \sigma)}{N}\left(i-\frac{N}{8}\right), & \frac{N}{8}<i \leq \frac{3 N}{8}, \\ d-\sigma+\frac{\sigma-\frac{2 \sigma}{N^{2}}}{\frac{N}{8}-2}\left(i-\frac{3 N}{8}\right), & \frac{3 N}{8}<i \leq \frac{N}{2}-2, \\ d+\frac{\sigma}{N^{2}}\left(i-\frac{N}{2}\right), & \frac{N}{2}-1 \leq i \leq \frac{N}{2}+1, \\ d+\frac{2 \sigma}{N^{2}}+\frac{\sigma-\frac{2 \sigma}{N^{2}}}{\frac{N}{8}-2}\left(i-\frac{N}{2}-2\right), & \frac{N}{2}+2 \leq i \leq \frac{5 N}{8} \\ d+\sigma+\frac{4(1-d-2 \sigma)}{N}\left(i-\frac{5 N}{8}\right), & \frac{5 N}{8}<i \leq \frac{7 N}{8} \\ 1-\sigma+\frac{8 \sigma}{N}\left(i-\frac{7 N}{8}\right), & \frac{7 N}{8}<i \leq N .\end{cases}
$$

Thus the mesh widths $h_{i}=x_{i}-x_{i-1}$ for $1 \leq i \leq N$ satisfy

$$
h_{i}= \begin{cases}h^{(1)}=\frac{8 \sigma}{N}, & 0<i \leq \frac{N}{8}, \frac{7 N}{8}<i \leq N \\ H^{(1)}=\frac{4(d-2 \sigma)}{N}, & \frac{N}{8}<i \leq \frac{3 N}{8} \\ h^{(2)}=\frac{\sigma-\frac{2 \sigma}{N^{2}}}{\frac{N}{8}-2}, & \frac{3 N}{8}<i \leq \frac{N}{2}-2, \frac{N}{2}+2<i \leq \frac{5 N}{8} \\ h^{(3)}=\frac{\sigma}{N^{2}}, & \frac{N}{2}-1 \leq i \leq \frac{N}{2}+2 \\ H^{(2)}=\frac{4(1-d-2 \sigma)}{N}, & \frac{5 N}{8}<i \leq \frac{7 N}{8}\end{cases}
$$

Here we have used an additional refined mesh at the region near $x=d$ for treating the lack of smoothness of the exact solution.

To approximate the solution of problem (1.1)-(1.2), we use a hybrid finite difference scheme on the Shishkin-type mesh $\bar{\Omega}^{N}=\left\{x_{i}\right\}_{0}^{N}$ :

$$
\begin{aligned}
& L_{H}^{N} U_{i}=f_{H, i}, \\
& U_{0}=A, \quad U_{N}=B,
\end{aligned}
$$

where

$$
\begin{aligned}
& L_{H}^{N} U_{i}= \begin{cases}-\varepsilon^{2} \delta^{2} U_{i}+\Gamma(b U)_{i}, & 1 \leq i<N, i \neq \frac{N}{2}, \\
-\varepsilon^{2} \delta^{2} U_{i}+\frac{b_{i-1} U_{i-1}+b_{i+1} U_{i+1}}{2}, & i=\frac{N}{2},\end{cases} \\
& f_{H, i}= \begin{cases}\Gamma f_{i}, & 1 \leq i<N, i \neq \frac{N}{2}, \\
\frac{f_{i-1}+f_{i+1}}{2}, & i=\frac{N}{2},\end{cases}
\end{aligned}
$$

and

$$
\begin{aligned}
& \delta^{2} U_{i}=\frac{1}{\hbar_{i}}\left(\frac{U_{i+1}-U_{i}}{h_{i+1}}-\frac{U_{i}-U_{i-1}}{h_{i}}\right), \\
& \Gamma U_{i}=\frac{1-\gamma_{i}^{-}}{6} U_{i-1}+\frac{4+\gamma_{i}^{-}+\gamma_{i}^{+}}{6} U_{i}+\frac{1-\gamma_{i}^{+}}{6} U_{i+1}, \\
& \gamma_{i}^{-}=\frac{h_{i+1}^{2}}{2 h_{i} \hbar_{i}}, \quad \gamma_{i}^{+}=\frac{h_{i}^{2}}{2 h_{i+1} \hbar_{i}}, \quad \hbar_{i}=\frac{h_{i}+h_{i+1}}{2} .
\end{aligned}
$$


This difference scheme is a combination of the Numerov scheme on the Shishkin-type mesh except for the point of discontinuity $x=d$ and the second-order difference schemes with the additional refined mesh for $x=d$, which is a modification of the difference scheme in [11-14]. We prove that the scheme is maximum-norm stable and has accuracy $O\left(\left(N^{-1} \ln N\right)^{4}\right)$ in the discrete maximum norm, independent of perturbation parameter.

\section{Analysis of the method}

\subsection{Stability analysis}

It is easy to check that the matrix associated with the discrete operator $L_{H}^{N}$ is not an Mmatrix. Hence the hybrid difference scheme (3.4)-(3.5) does not satisfy the discrete maximum principle. However, a maximum-norm stability analysis can be conducted. The technique used in this paper to analyze the stability of the hybrid discrete scheme is similar to the method in [12].

Define the new difference operator $\Lambda$ as follows:

$$
\Lambda y_{i}= \begin{cases}-\varepsilon^{2} \delta^{2} y_{i}-\frac{\gamma_{i}^{-}}{6} b_{i} y_{i-1}+\frac{4+\gamma_{i}^{-}+\gamma_{i}^{+}}{6} b_{i} y_{i}-\frac{\gamma_{i}^{+}}{6} b_{i} y_{i+1}, & 1 \leq i<N, i \neq \frac{N}{2} \\ -\varepsilon^{2} \delta^{2} y_{i}+\frac{b_{i-1} y_{i-1}+b_{i+1} y_{i+1}}{2}, & i=\frac{N}{2}\end{cases}
$$

We will prove that the operator $\Lambda$ satisfies the following discrete maximum principle.

Lemma 4.1 (Discrete maximum principle) The operator defined in (4.1) satisfies a discrete maximum principle for sufficiently large $N$, that is, if $y$ is a mesh function satisfying $y_{0} \geq 0$, $y_{N} \geq 0$, and $\Lambda y_{i} \geq 0$ for $1 \leq i<N$, then $y_{i} \geq 0$ for all $i$.

Proof It is easy to verify that the matrix associated with $\Lambda$ has positive diagonal entries, nonpositive off-diagonal, and positive row-sum for sufficiently large $N$. Therefore, the matrix associated with $\Lambda$ is an M-matrix. From this we conclude that the lemma holds.

Dividing (4.1) by $b_{i}$ and applying the discrete maximum principle, we can obtain

$$
\|y\|_{\bar{\Omega}^{N}} \leq \frac{3}{2}\left\|\frac{\Lambda y}{b}\right\|_{\bar{\Omega}^{N}}
$$

for any mesh function $y$ with $y_{0}=y_{N}=0$, which will be used to establish the stability of the operator $L_{H}^{N}$.

Lemma 4.2 (Stability) There exists a constant $\kappa \in(0,1)$ such that, for sufficiently large $N$,

$$
\|y\|_{\bar{\Omega}^{N}} \leq \frac{3}{1-\kappa}\left\|\frac{L_{H}^{N} y}{b}\right\|_{\bar{\Omega}^{N}}
$$

for any mesh function $y$ with $y_{0}=y_{N}=0$.

Proof Combining (3.6) and (4.1), we get

$$
\Lambda y_{i}=L_{H}^{N} y_{i}-\frac{b_{i-1}}{6} y_{i-1}-\frac{b_{i+1}}{6} y_{i+1}+\frac{\gamma_{i}^{-}}{6}\left(b_{i-1}-b_{i}\right) y_{i-1}+\frac{\gamma_{i}^{+}}{6}\left(b_{i+1}-b_{i}\right) y_{i+1}
$$


for $1 \leq i<\frac{N}{2}$ and $\frac{N}{2}<i<N$. Hence we have

$$
\left|\Lambda y_{i}\right| \leq\left|L_{H}^{N} y_{i}\right|+\left(\frac{b_{i-1}+b_{i+1}}{6}+\frac{h_{i}+h_{i+1}}{6}\left\|b^{\prime}\right\|_{\bar{\Omega}}\right)\|y\|_{\bar{\Omega}^{N}}
$$

for $1 \leq i<\frac{N}{2}$ and $\frac{N}{2}<i<N$. Since $b$ is a sufficiently smooth function on $\bar{\Omega}$, there exists a constant $\kappa \in(0,1)$ such that

$$
\frac{b_{i-1}+b_{i+1}}{6}+\frac{h_{i}+h_{i+1}}{6}\left\|b^{\prime}\right\|_{\bar{\Omega}} \leq \frac{1+\kappa}{3} b_{i}
$$

for sufficiently large $N$ independent $\varepsilon$. Therefore,

$$
\left|\Lambda y_{i}\right| \leq \begin{cases}\left|L_{H}^{N} y_{i}\right|+\frac{1+\kappa}{3} b_{i}\|y\|_{\Omega^{N}}, & 1 \leq i<N, i \neq \frac{N}{2} \\ \left|L_{H}^{N} y_{i}\right|, & i=\frac{N}{2} .\end{cases}
$$

Substituting (4.3) into inequality (4.2), we obtain

$$
\|y\|_{\bar{\Omega}^{N}} \leq \frac{3}{2}\left\|\frac{L_{H}^{N} y}{b}\right\|_{\bar{\Omega}^{N}}+\frac{1+\kappa}{2}\|y\|_{\bar{\Omega}^{N}} .
$$

From this inequality we get the desired result.

\subsection{Error analysis}

Let $z_{i}=U_{i}-u_{i}$, where $U_{i}$ is the solution of problem (3.4)-(3.5), and $u_{i}$ is the solution of problem (1.1)-(1.2) at mesh point $x_{i}$. Then the error $z$ satisfies the following discrete problem:

$$
\begin{aligned}
& L_{H}^{N} z_{i}=R_{i}, \quad 1 \leq i<N, \\
& z_{0}=z_{N}=0,
\end{aligned}
$$

where

$$
R_{i}= \begin{cases}-\varepsilon^{2}\left(\Gamma u_{i}^{\prime \prime}-\delta^{2} u_{i}\right), & 1 \leq i<N, i \neq \frac{N}{2}, \\ -\varepsilon^{2}\left[\frac{1}{2}\left(u_{i-1}^{\prime \prime}+u_{i+1}^{\prime \prime}\right)-\delta^{2} u_{i}\right], & i=N / 2 .\end{cases}
$$

The error $z$ of the discrete scheme can be decomposed as follows:

$$
z=\varphi+\psi
$$

where $\varphi$ is the solution of problem

$$
\Lambda \varphi_{i}=L_{H}^{N} z_{i}=R_{i}, \quad 1 \leq i<N
$$

and $\psi$ is the solution of problem

$$
\Lambda \psi_{i}= \begin{cases}-\frac{b_{i-1}}{6} z_{i-1}+\frac{\gamma_{i}^{-}}{6}\left(b_{i-1}-b_{i}\right) z_{i-1}, & \\ -\frac{b_{i+1}}{6} z_{i+1}+\frac{\gamma_{i}^{+}}{6}\left(b_{i+1}-b_{i}\right) z_{i+1}, & 1 \leq i<N, i \neq \frac{N}{2}, \\ 0, & i=\frac{N}{2} .\end{cases}
$$


Using the same method as in the stability analysis, we can obtain

$$
\left|\Lambda \psi_{i}\right| \leq \frac{1+\kappa}{3} b_{i}\|z\|_{\bar{\Omega}^{N}}, \quad 1 \leq i<N
$$

for sufficiently large $N$ independent of $\varepsilon$, where $\kappa \in(0,1)$ is a positive constant. Applying the stability inequality (4.2), we have

$$
\|\psi\|_{\bar{\Omega}^{N}} \leq \frac{1+\kappa}{2}\|z\|_{\bar{\Omega}^{N}}
$$

Combining (4.7) with (4.10), we obtain

$$
\|z\|_{\bar{\Omega}^{N}} \leq\|\varphi\|_{\bar{\Omega}^{N}}+\|\psi\|_{\bar{\Omega}^{N}} \leq\|\varphi\|_{\bar{\Omega}^{N}}+\frac{1+\kappa}{2}\|z\|_{\bar{\Omega}^{N}},
$$

that is,

$$
\|z\|_{\bar{\Omega}^{N}} \leq \frac{2}{1-\kappa}\|\varphi\|_{\bar{\Omega}^{N}} .
$$

Hence, for estimating the error $z$, we are left with estimating $\varphi$.

The following lemma gives us a useful formula for the truncation error.

Lemma 4.3 Let $g \in C^{6}\left[x_{i-1}, x_{i+1}\right]$. Then we have the following estimates:

$$
\left|\Gamma g_{i}^{\prime \prime}-\delta^{2} g_{i}\right| \leq \begin{cases}C\left(h_{i}+h_{i+1}\right)^{3}\left\|g^{(5)}\right\|_{\left[x_{i-1}, x_{i+1}\right]}, & \\ C\left\|g^{\prime \prime}\right\|_{\left[x_{i-1}, x_{i+1}\right]}, & \text { if } h_{i}=h_{i+1}, \\ C h_{i}^{4}\left\|g^{(6)}\right\|_{\left[x_{i-1}, x_{i+1}\right]}, & \text { if } h_{i}=h_{i+1}, \\ C\left\|g^{\prime \prime}\right\|_{\left[x_{i}, x_{i+1}\right]}+C \gamma_{i}^{-} h_{i}\left\|g^{\prime \prime \prime}\right\|_{\left[x_{i-1}, x_{i}\right]}, & \text { if } h_{i} \leq h_{i+1}, \\ C\left\|g^{\prime \prime}\right\|_{\left[x_{i-1}, x_{i}\right]}+C \gamma_{i}^{+} h_{i+1}\left\|g^{\prime \prime \prime}\right\|_{\left[x_{i}, x_{i+1}\right]}, & \text { if } h_{i} \geq h_{i+1} .\end{cases}
$$

Proof It is easy to get the desired results by using the Taylor expansions given in [12].

The next lemma gives us the truncation error estimates.

Lemma 4.4 Under assumption (3.1), we have the following bounds of the truncation error $R$ for the difference scheme (3.4)-(3.5):

$$
\left|R_{i}\right| \leq \begin{cases}C\left(N^{-1} \ln N\right)^{4}, & i \in \Theta \backslash \theta, \\ C\left(N^{-1} \ln N\right)^{3}, & i \in\left\{\frac{N}{2}-2, \frac{N}{2}+2\right\}, \\ C N^{-2} \ln N, & i \in\left\{\frac{N}{2}\right\}, \\ C N^{-4}+C \varepsilon N^{-3}+C \gamma_{i}^{-} N^{-5} \ln N, & i \in\left\{\frac{N}{8}, \frac{5 N}{8}\right\}, \\ C N^{-4}+C \varepsilon N^{-3}+C \gamma_{i}^{+} N^{-5} \ln N, & i \in\left\{\frac{3 N}{8}, \frac{7 N}{8}\right\},\end{cases}
$$

where $\Theta=\{i \mid 1 \leq i<N\}$ and $\theta=\left\{\frac{N}{8}, \frac{3 N}{8}, \frac{N}{2}-2, \frac{N}{2}, \frac{N}{2}+2, \frac{5 N}{8}, \frac{7 N}{8}\right\}$. 
Proof It is easy to verify that

$$
h_{i} \leq \begin{cases}C \varepsilon N^{-1} \ln N, & 1 \leq i \leq \frac{N}{8}, \frac{3 N}{8}<i \leq \frac{N}{2}-2, \frac{N}{2}+2<i \leq \frac{5 N}{8}, \frac{7 N}{8}<i \leq N \\ C N^{-1}, & \frac{N}{8}<i \leq \frac{3 N}{8}, \frac{5 N}{8}<i \leq \frac{7 N}{8} \\ C \varepsilon N^{-2} \ln N, & \frac{N}{2}-1 \leq i \leq \frac{N}{2}+2 .\end{cases}
$$

(1) For $x_{i} \in(0, \sigma) \cup(1-\sigma, 1)$, using the third bound of (4.12), the mesh widths (4.14), and the bounds of the derivatives for the exact solution in Lemma 2.1, we have

$$
\begin{aligned}
\left|R_{i}\right| & =\varepsilon^{2}\left|\Gamma u_{i}^{\prime \prime}-\delta^{2} u_{i}\right| \\
& \leq C \varepsilon^{2} h_{i}^{4}\left\|u^{(6)}\right\|_{\left[x_{i-1}, x_{i+1}\right]} \\
& \leq C \varepsilon^{-4}\left(h^{(1)}\right)^{4} \\
& \leq C\left(N^{-1} \ln N\right)^{4}, \quad 1 \leq i<\frac{N}{8}, \frac{7 N}{8}<i<N .
\end{aligned}
$$

(2) For $x_{i} \in(\sigma, d-\sigma) \cup(d+\sigma, 1-\sigma)$, recalling the decomposition of $u$ in (2.1), we have

$$
\left|R_{i}\right|=\varepsilon^{2}\left|\Gamma u_{i}^{\prime \prime}-\delta^{2} u_{i}\right| \leq \varepsilon^{2}\left|\Gamma v_{i}^{\prime \prime}-\delta^{2} v_{i}\right|+\varepsilon^{2}\left|\Gamma w_{i}^{\prime \prime}-\delta^{2} w_{i}\right|
$$

For the first term in the right-hand side of (4.16), using the third bound of (4.12), the mesh widths (4.14), and the bound of the regular part $v$ in (2.2) we get

$$
\varepsilon^{2}\left|\Gamma v_{i}^{\prime \prime}-\delta^{2} v_{i}\right| \leq C N^{-4}, \quad \frac{N}{8}<i<\frac{3 N}{8}, \frac{5 N}{8}<i<\frac{7 N}{8} .
$$

For the second term in the right-hand side in (4.16), using the second bound of (4.12) and the bound of the layer part $w$ in (2.3) we obtain

$$
\varepsilon^{2}\left|\Gamma w_{i}^{\prime \prime}-\delta^{2} w_{i}\right| \leq C N^{-4}, \quad \frac{N}{8}<i<\frac{3 N}{8}, \frac{5 N}{8}<i<\frac{7 N}{8} .
$$

Therefore, substituting (4.17)-(4.18) into (4.16), we have

$$
\left|R_{i}\right| \leq C N^{-4}, \quad \frac{N}{8}<i<\frac{3 N}{8}, \frac{5 N}{8}<i<\frac{7 N}{8} .
$$

(3) For $x_{i} \in\left(d-\sigma, d-\frac{2 \sigma}{N^{2}}\right) \cup\left(d+\frac{2 \sigma}{N^{2}}, d+\sigma\right) \cup\left\{d-\frac{\sigma}{N^{2}}, d+\frac{\sigma}{N^{2}}\right\}$, using the third bound of (4.12), the mesh widths (4.14), and the bounds of the derivatives for the exact solution in Lemma 2.1, we have

$$
\begin{aligned}
\left|R_{i}\right| & =\varepsilon^{2}\left|\Gamma u_{i}^{\prime \prime}-\delta^{2} u_{i}\right| \\
& \leq C \varepsilon^{2} h_{i}^{4}\left\|u^{(6)}\right\|_{\left[x_{i-1}, x_{i+1}\right]} \\
& \leq C\left(N^{-1} \ln N\right)^{4}
\end{aligned}
$$

for $\frac{3 N}{8}<i<\frac{N}{2}-2, \frac{N}{2}+2<i<\frac{5 N}{8}$, and $i=\frac{N}{2}-1, \frac{N}{2}+1$. 
(4) For $x_{i} \in\left\{d-\frac{2 \sigma}{N^{2}}, d+\frac{2 \sigma}{N^{2}}\right\}$, using the first bound of (4.12), the mesh widths (4.14), and the bounds of the derivatives for the exact solution in Lemma 2.1, we have

$$
\begin{aligned}
\left|R_{i}\right| & =\varepsilon^{2}\left|\Gamma u_{i}^{\prime \prime}-\delta^{2} u_{i}\right| \leq C \varepsilon^{2}\left(h_{i}+h_{i+1}\right)^{3}\left\|u^{(5)}\right\|_{\left[x_{i-1}, x_{i+1}\right]} \\
& \leq C\left(N^{-1} \ln N\right)^{3}, \quad i=\frac{N}{2}-2, \frac{N}{2}+2 .
\end{aligned}
$$

(5) For $x_{i} \in\{d\}$, we also apply the Taylor expansion about $x=x_{i}$ to get

$$
\begin{aligned}
\left|R_{i}\right|= & \mid-\frac{1}{2} \varepsilon^{2}\left(u_{i-1}^{\prime \prime}-\delta^{2} u_{i-1}\right)-\frac{1}{2} \varepsilon^{2}\left(u_{i+1}^{\prime \prime}-\delta^{2} u_{i+1}\right) \\
& +\frac{\varepsilon^{2}}{h^{(3)}}\left(\frac{-u_{i-2}+4 u_{i-1}-3 u_{i}}{2 h^{(3)}}-\frac{u_{i+2}-4 u_{i+1}+3 u_{i}}{2 h^{(3)}}\right) \mid \\
\leq & \frac{1}{2} \varepsilon^{2}\left|u_{i-1}^{\prime \prime}-\delta^{2} u_{i-1}\right|+\frac{1}{2} \varepsilon^{2}\left|u_{i+1}^{\prime \prime}-\delta^{2} u_{i+1}\right| \\
& +\frac{\varepsilon^{2}}{h^{(3)}}\left|\frac{-u_{i-2}+4 u_{i-1}-3 u_{i}}{2 h^{(3)}}-u_{i}^{\prime}\right|+\frac{\varepsilon^{2}}{h^{(3)}}\left|\frac{u_{i+2}-4 u_{i+1}+3 u_{i}}{2 h^{(3)}}-u_{i}^{\prime}\right| \\
\leq & C \varepsilon^{-2}\left(h^{(3)}\right)^{2}+C \varepsilon^{-1} h^{(3)} \\
\leq & C N^{-2} \ln N, \quad i=\frac{N}{2},
\end{aligned}
$$

where we also have used Lemma 2.1 and the mesh widths (4.14).

(6) For $x_{i} \in\{\sigma, 1-\sigma, d-\sigma, d+\sigma\}$, we also decompose the truncation error into two parts:

$$
\left|R_{i}\right|=\varepsilon^{2}\left|\Gamma u_{i}^{\prime \prime}-\delta^{2} u_{i}\right| \leq \varepsilon^{2}\left|\Gamma v_{i}^{\prime \prime}-\delta^{2} v_{i}\right|+\varepsilon^{2}\left|\Gamma w_{i}^{\prime \prime}-\delta^{2} w_{i}\right|
$$

For the first term in the right-hand side in (4.23), using the first bound of (4.12), the mesh widths (4.14), and the bound of the regular part $v$ in (2.2), we get

$$
\varepsilon^{2}\left|\Gamma v_{i}^{\prime \prime}-\delta^{2} v_{i}\right| \leq C \varepsilon N^{-3}, \quad i=\frac{N}{8}, \frac{3 N}{8}, \frac{5 N}{8}, \frac{7 N}{8} .
$$

For the second term in the right-hand side in (4.23), using the fourth bound of (4.12) and the bound of the layer part $w$ in (2.3), we obtain

$$
\begin{aligned}
\varepsilon^{2}\left|\Gamma w_{i}^{\prime \prime}-\delta^{2} w_{i}\right| \leq & C\left(e^{-\beta x_{i} / \varepsilon}+e^{-\beta\left(1-x_{N-i}\right) / \varepsilon}\right) \\
& +C \gamma_{i}^{-} N^{-1} \ln N\left(e^{-\beta x_{i-1} / \varepsilon}+e^{-\beta\left(1-x_{N-i+1}\right) / \varepsilon}\right) \\
\leq & C N^{-4}+C \gamma_{i}^{-} N^{-5} \ln N, \quad i=\frac{N}{8}, \frac{5 N}{8}
\end{aligned}
$$

By applying the analogous methods used in estimating (4.25) we can obtain

$$
\varepsilon^{2}\left|\Gamma w_{i}^{\prime \prime}-\delta^{2} w_{i}\right| \leq C N^{-4}+C \gamma_{i}^{+} N^{-5} \ln N, \quad i=\frac{3 N}{8}, \frac{7 N}{8},
$$


where the bound of the truncation error is replaced by the fifth bound of (4.12). Hence, substituting (4.24)-(4.26) into (4.23), we have

$$
\left|R_{i}\right| \leq \begin{cases}C N^{-4}+C \varepsilon N^{-3}+C \gamma_{i}^{-} N^{-5} \ln N, & i=\frac{N}{8}, \frac{5 N}{8}, \\ C N^{-4}+C \varepsilon N^{-3}+C \gamma_{i}^{+} N^{-5} \ln N, & i=\frac{3 N}{8}, \frac{7 N}{8} .\end{cases}
$$

Therefore, from (4.15), (4.19)-(4.22), and (4.27) we conclude that the lemma holds.

Now we can derive our main result for the hybrid difference scheme.

Theorem 4.5 Let $u$ be the solution of problem (1.1)-(1.2), and $U$ be the solution of finite difference scheme (3.4)-(3.5) on the Shishkin-type mesh (3.2). Then, under assumption (3.1), we have the following error estimate:

$$
\|U-u\|_{\bar{\Omega}^{N}} \leq C\left(N^{-1} \ln N\right)^{4}
$$

for sufficiently large $N$, where $C$ is a positive constant independent of $\varepsilon$ and the mesh.

Proof From (4.11) we know that estimating the error $U-u$ is equivalent to estimating the bound of $\varphi$, which can be done by using a barrier function technique. Following the idea in [12], we define a mesh function as follows:

$$
\chi_{i}= \begin{cases}\frac{x_{i}}{\sigma}, & 0 \leq i \leq \frac{N}{8}, \\ 1, & \frac{N}{8}<i \leq \frac{3 N}{8}, \\ \frac{d-2 h^{(3)}-h^{(2)}-x_{i}}{\sigma}, & \frac{3 N}{8}+1 \leq i \leq \frac{N}{2}-3, \\ \frac{\sigma+h^{(3)}}{\sigma}, & \frac{N}{2}-2 \leq i \leq \frac{N}{2}, \\ \frac{d+\sigma+h^{(3)}-x_{i}}{\sigma}, & \frac{N}{2}<i \leq \frac{N}{2}+2, \\ \frac{x_{i}-d}{\sigma}, & \frac{N}{2}+3 \leq i \leq \frac{5 N}{8} \\ 1, & \frac{5 N}{8}<i \leq \frac{7 N}{8} \\ \frac{1-x_{i}}{\sigma}, & \frac{7 N}{8}<i \leq N .\end{cases}
$$

Consider the discrete barrier function

$$
W_{i}=C\left(1+\chi_{i}\right)\left(N^{-1} \ln N\right)^{4}
$$

where $C$ is a positive constant independent of $\varepsilon$ and the mesh. By a direct calculation we get

$$
\Lambda W_{i} \geq R_{i}, \quad 1 \leq i<N
$$

for sufficiently large $N$. Hence, applying the discrete maximum principle (Lemma 4.1) to $W \pm \varphi$ on $\bar{\Omega}^{N}$, we have

$$
\|\varphi\|_{\bar{\Omega}^{N}} \leq C\left(N^{-1} \ln N\right)^{4} .
$$

Combining (4.31) with (4.11) completes the proof. 


\section{Numerical experiments}

In this section, we verify experimentally the theoretical results obtained in the preceding section. The error estimates and convergence rates for the hybrid difference scheme are presented for two examples presented in [2].

Example 5.1 Consider the reaction-diffusion problem

$$
\begin{aligned}
& -\varepsilon^{2} u^{\prime \prime}(x)+u(x)=f(x), \quad x \in \Omega^{-} \cup \Omega^{+}, \\
& u(0)=u(1)=f(0),
\end{aligned}
$$

where

$$
f(x)= \begin{cases}-0.5 x, & 0 \leq x \leq 0.5 \\ 0.5, & 0.5<x \leq 1\end{cases}
$$

The exact solution of this example is

$$
u(x)= \begin{cases}0.25(\xi+\eta)\left(e^{-(0.5-x) / \varepsilon}-e^{-(0.5+x) / \varepsilon}\right)-0.5 x, & 0 \leq x \leq 0.5, \\ 0.25(\xi-\eta) e^{-1 /(2 \varepsilon)}\left(e^{-(x-1) / \varepsilon}-e^{-(1-x) / \varepsilon}\right)+0.5\left(1-e^{-(1-x) / \varepsilon}\right), & 0.5<x \leq 1,\end{cases}
$$

where the constants $\xi$ and $\eta$ are

$$
\begin{aligned}
\xi & =\frac{\varepsilon-e^{-1 /(2 \varepsilon)}}{1+e^{-1 / \varepsilon}}, \\
\eta & =\frac{1.5-e^{-1 /(2 \varepsilon)}}{1-e^{-1 / \varepsilon}} .
\end{aligned}
$$

We measure the accuracy in the discrete maximum norm

$$
e_{\varepsilon}^{N}=\max _{1 \leq i \leq N}\left|u_{\varepsilon, i}-U_{\varepsilon, i}\right|, \quad E^{N}=\max _{\varepsilon} e_{\varepsilon}^{N}
$$

and the 'Shishkin' convergence rate

$$
\begin{aligned}
& r_{\varepsilon}^{N}=\frac{\ln e_{\varepsilon}^{N}-\ln e_{\varepsilon}^{2 N}}{\ln (2 \ln N)-\ln (\ln (2 N))}, \\
& R^{N}=\frac{\ln E^{N}-\ln E^{2 N}}{\ln (2 \ln N)-\ln (\ln (2 N))} .
\end{aligned}
$$

Numerical results for Example 5.1 are listed in Table 1.

Example 5.2 Consider the reaction-diffusion problem

$$
\begin{aligned}
& -\varepsilon^{2} u^{\prime \prime}(x)+b(x) u(x)=f(x), \quad x \in \Omega^{-} \cup \Omega^{+}, \\
& u(0)=u(1)=f(0),
\end{aligned}
$$


Table 1 Error estimates and convergence rates for various $N$ for Example 5.1

\begin{tabular}{lllllll}
\hline \multicolumn{6}{c}{ Number of mesh points $\boldsymbol{N}$} & \multicolumn{5}{l}{} \\
\hline$\varepsilon$ & 64 & 128 & 256 & 512 & 1,024 & 2,048 \\
$10^{-2}$ & $3.4894 \mathrm{e}-3$ & $1.7662 \mathrm{e}-4$ & $8.7528 \mathrm{e}-6$ & $5.5665 \mathrm{e}-7$ & $3.4830 \mathrm{e}-8$ & $2.1775 \mathrm{e}-9$ \\
& 5.535 & 5.369 & 4.789 & 4.715 & 4.637 & - \\
$10^{-3}$ & $7.3009 \mathrm{e}-3$ & $9.4205 \mathrm{e}-4$ & $8.1893 \mathrm{e}-5$ & $8.6906 \mathrm{e}-6$ & $8.3930 \mathrm{e}-7$ & $7.7064 \mathrm{e}-8$ \\
& 3.799 & 4.365 & 3.899 & 3.977 & 3.994 & - \\
$10^{-4}$ & $7.2974 \mathrm{e}-3$ & $9.4202 \mathrm{e}-4$ & $8.1893 \mathrm{e}-5$ & $8.6906 \mathrm{e}-6$ & $8.3930 \mathrm{e}-7$ & $7.7064 \mathrm{e}-8$ \\
& 3.798 & 4.365 & 3.899 & 3.977 & 3.994 & - \\
$10^{-5}$ & $7.2970 \mathrm{e}-3$ & $9.4220 \mathrm{e}-4$ & $8.1893 \mathrm{e}-5$ & $8.6906 \mathrm{e}-6$ & $8.3930 \mathrm{e}-7$ & $7.7064 \mathrm{e}-8$ \\
& 3.798 & 4.365 & 3.899 & 3.977 & 3.994 & - \\
$10^{-6}$ & $7.2970 \mathrm{e}-3$ & $9.4202 \mathrm{e}-4$ & $8.1893 \mathrm{e}-5$ & $8.6906 \mathrm{e}-6$ & $8.3930 \mathrm{e}-7$ & $7.7064 \mathrm{e}-8$ \\
& 3.798 & 4.365 & 3.899 & 3.977 & 3.994 & - \\
$10^{-7}$ & $7.2970 \mathrm{e}-3$ & $9.4202 \mathrm{e}-4$ & $8.1893 \mathrm{e}-5$ & $8.6906 \mathrm{e}-6$ & $8.3930 \mathrm{e}-7$ & $7.7064 \mathrm{e}-8$ \\
& 3.798 & 4.365 & 3.899 & 3.977 & 3.994 & - \\
$10^{-8}$ & $7.2970 \mathrm{e}-3$ & $9.4202 \mathrm{e}-4$ & $8.1893 \mathrm{e}-5$ & $8.6906 \mathrm{e}-6$ & $8.3929 \mathrm{e}-7$ & $7.7064 \mathrm{e}-8$ \\
& 3.798 & 4.365 & 3.899 & 3.977 & 3.994 & - \\
$10^{-9}$ & $7.2970 \mathrm{e}-3$ & $9.4202 \mathrm{e}-4$ & $8.1893 \mathrm{e}-5$ & $8.6906 \mathrm{e}-6$ & $8.3930 \mathrm{e}-7$ & $7.7064 \mathrm{e}-8$ \\
& 3.798 & 4.365 & 3.899 & 3.977 & 3.994 & - \\
$E^{N}$ & $7.3009 \mathrm{e}-3$ & $9.4205 \mathrm{e}-4$ & $8.1893 \mathrm{e}-5$ & $8.6906 \mathrm{e}-6$ & $8.3930 \mathrm{e}-7$ & $7.7064 \mathrm{e}-8$ \\
$R^{N}$ & 3.798 & 4.365 & 3.899 & 3.977 & 3.994 & - \\
\hline
\end{tabular}

where

$$
\begin{aligned}
& b(x)= \begin{cases}2 x+1, & 0 \leq x \leq 0.5, \\
2(1-x)+1, & 0.5<x \leq 1,\end{cases} \\
& f(x)= \begin{cases}-0.5, & 0 \leq x \leq 0.5 \\
0.5, & 0.5<x \leq 1\end{cases}
\end{aligned}
$$

The exact solution of this example is not available. Therefore, we use the double mesh principle to estimate the errors and compute the experiment convergence rates. Because mesh points for $N$ and $2 N$ do not match, we use the piecewise cubic spline interpolation to get the solution for $2 N$. That is, $\bar{U}_{\varepsilon}^{2 N}(x)$ is a piecewise cubic spline interpolation of the approximated solution $U_{\varepsilon}^{2 N}$, and $\bar{U}_{\varepsilon}^{2 N}\left(x_{i}\right)$ is the value of the function $\bar{U}_{\varepsilon}^{2 N}(x)$ at mesh point $x_{i}$ for $N$. We measure the accuracy in the discrete maximum norm

$$
e_{\varepsilon}^{N}=\max _{1 \leq i \leq N}\left|U_{\varepsilon, i}^{N}-\bar{U}_{\varepsilon}^{2 N}\left(x_{i}\right)\right|, \quad E^{N}=\max _{\varepsilon} e_{\varepsilon}^{N}
$$

and the 'Shishkin' convergence rate

$$
\begin{aligned}
& r_{\varepsilon}^{N}=\frac{\ln e_{\varepsilon}^{N}-\ln e_{\varepsilon}^{2 N}}{\ln (2 \ln N)-\ln (\ln (2 N))}, \\
& R^{N}=\frac{\ln E^{N}-\ln E^{2 N}}{\ln (2 \ln N)-\ln (\ln (2 N))} .
\end{aligned}
$$

Numerical results for Example 5.2 are listed in Table 2.

From Tables 1 and 2 we see that the results seem to be $\varepsilon$-uniform as expected and $R^{N}$ are close to 4 for sufficiently large $N$ independent of $\varepsilon$, which supports the convergence estimate of Theorem 4.5. They indicate that the theoretical results are fairly sharp. 
Table 2 Error estimates and convergence rates for various $N$ for Example 5.2

\begin{tabular}{lllllll}
\hline Number of mesh points $\boldsymbol{N}$ & \multicolumn{5}{l}{} \\
\hline$\varepsilon$ & 64 & 128 & 256 & 512 & 1,024 & 2,048 \\
$10^{-2}$ & $5.5036 \mathrm{e}-3$ & $4.3650 \mathrm{e}-4$ & $2.1633 \mathrm{e}-5$ & $1.1995 \mathrm{e}-6$ & $7.0962 \mathrm{e}-8$ & $4.2920 \mathrm{e}-9$ \\
& 4.702 & 5.369 & 5.027 & 4.811 & 4.693 & - \\
$10^{-3}$ & $9.0903 \mathrm{e}-3$ & $1.7499 \mathrm{e}-3$ & $1.9075 \mathrm{e}-4$ & $1.6259 \mathrm{e}-5$ & $1.4964 \mathrm{e}-6$ & $1.4532 \mathrm{e}-7$ \\
& 3.057 & 3.960 & 4.280 & 4.059 & 3.901 & - \\
$10^{-4}$ & $9.0667 \mathrm{e}-3$ & $1.7458 \mathrm{e}-3$ & $1.9024 \mathrm{e}-4$ & $1.6213 \mathrm{e}-5$ & $1.5079 \mathrm{e}-6$ & $1.4493 \mathrm{e}-7$ \\
& 3.057 & 3.961 & 4.280 & 4.041 & 3.918 & - \\
$10^{-5}$ & $9.0644 \mathrm{e}-3$ & $1.7454 \mathrm{e}-3$ & $1.9019 \mathrm{e}-4$ & $1.6208 \mathrm{e}-5$ & $1.5091 \mathrm{e}-6$ & $1.4489 \mathrm{e}-7$ \\
& 3.056 & 3.961 & 4.280 & 4.039 & 3.920 & - \\
$10^{-6}$ & $9.0641 \mathrm{e}-3$ & $1.7453 \mathrm{e}-3$ & $1.9018 \mathrm{e}-4$ & $1.6208 \mathrm{e}-5$ & $1.5092 \mathrm{e}-6$ & $1.4489 \mathrm{e}-7$ \\
& 3.056 & 3.961 & 4.280 & 4.039 & 3.920 & - \\
$10^{-7}$ & $9.0641 \mathrm{e}-3$ & $1.7453 \mathrm{e}-3$ & $1.9018 \mathrm{e}-4$ & $1.6208 \mathrm{e}-5$ & $1.5092 \mathrm{e}-6$ & $1.4491 \mathrm{e}-7$ \\
& 3.056 & 3.961 & 4.280 & 4.039 & 3.920 & - \\
$10^{-8}$ & $9.0641 \mathrm{e}-3$ & $1.7453 \mathrm{e}-3$ & $1.9018 \mathrm{e}-4$ & $1.6208 \mathrm{e}-5$ & $1.5092 \mathrm{e}-6$ & $1.4507 \mathrm{e}-7$ \\
& 3.056 & 3.961 & 4.280 & 4.039 & 3.918 & - \\
$10^{-9}$ & $9.0641 \mathrm{e}-3$ & $1.7453 \mathrm{e}-3$ & $1.9018 \mathrm{e}-4$ & $1.6209 \mathrm{e}-5$ & $1.5092 \mathrm{e}-6$ & $1.4666 \mathrm{e}-7$ \\
& 3.056 & 3.961 & 4.280 & 4.039 & 3.899 & - \\
$E^{N}$ & $9.0903 \mathrm{e}-3$ & $1.7499 \mathrm{e}-3$ & $1.9075 \mathrm{e}-4$ & $1.6259 \mathrm{e}-5$ & $1.5092 \mathrm{e}-6$ & $1.4666 \mathrm{e}-7$ \\
$R^{N}$ & 3.057 & 3.960 & 4.280 & 4.044 & 3.899 & - \\
\hline
\end{tabular}

\section{Conclusion}

In this paper, we presented a high-order finite difference method for solving a singularly perturbed reaction-diffusion problem with a discontinuous source term. This difference scheme is a combination of a nonequidistant generalization of the Numerov scheme on the Shishkin-type mesh except for the point of discontinuity and a second-order difference scheme on an additional refined mesh at the point of discontinuity. This hybrid difference scheme for the singularly perturbed reaction-diffusion problem with a discontinuous source term is a modification of the difference scheme used in [11-14] for the singularly perturbed reaction-diffusion problem with sufficiently smooth data. Although the difference scheme does not satisfy the discrete maximum principle, the maximum norm stability of the scheme is established. We have shown that the scheme has accuracy $O\left(\left(N^{-1} \ln N\right)^{4}\right)$ in the discrete maximum norm, independently of perturbation parameter. Numerical experiments support these theoretical results.

\section{Acknowledgements}

We would like to thank the anonymous reviewer for some suggestions for the improvement of this paper. The work was supported by Humanities and Social Sciences Planning Fund of Ministry of Education of China (Grant No. 14YJC790006), Zhejiang Province Natural Science Foundation (Grant No. Y17D010024), Ningbo Municipal Natural Science Foundation (Grant Nos. 2017A610131, 2017A610140, 2015A610161), Ningbo Municipal Soft Science Foundation (Grant No. 2015A10045) and Soft Science Project of Zhejiang Province (Grant No. 2015C35007).

Competing interests

The authors declare that there is no conflict of interests regarding the publication of this paper. The authors confirm that the mentioned received funding in the 'Acknowledgements' section does not lead to any conflict of interests regarding the publication of this manuscript.

Authors' contributions

All authors read and approved the final manuscript.

\section{Author details}

${ }^{1}$ College of Finance and Trade, Ningbo Dahongying University, Ningbo, China. ${ }^{2}$ Institute of Mathematics, Zhejiang Wanli University, Ningbo, China.

\section{Publisher's Note}

Springer Nature remains neutral with regard to jurisdictional claims in published maps and institutional affiliations. 
Received: 30 March 2017 Accepted: 7 July 2017 Published online: 24 July 2017

\section{References}

1. Chandru, M, Prabha, T, Shanthi, V: A hybrid difference scheme for a second-order singularly perturbed reaction-diffusion problem with non-smooth data. Int. J. Appl. Comput. Math. 1, 87-100 (2015)

2. Miller, JJH, O'Riordan, E, Shishkin, GI, Wang, S: A parameter-uniform Schwarz method for a singularly perturbed reaction-diffusion problem with an interior layer. Appl. Numer. Math. 35, 323-337 (2000)

3. Farrell, PA, Hegarty, AF, Miller, JJH, O'Riordan, E, Shishkin, Gl: Robust Computational Techniques for Boundary Layers. Chapman \& Hall/CRC Press, New York (2000)

4. Kadalbajoo, MK, Gupta, V: A brief survey on numerical methods for solving singularly perturbed problems. Appl. Math. Comput. 217, 3641-3716 (2010)

5. Roos, H-G, Zarin, H: A second-order scheme for singularly perturbed differential equations with discontinuous source term. J. Numer. Math. 10, 275-289 (2002)

6. Farrell, PA, O'Riordan, E, Shishkin, Gl: A class of singularly perturbed semilinear differential equations with interior layers. Math. Comput. 74, 1759-1776 (2005)

7. Boglaev, I, Pack, S: A uniformly convergent method for a singularly perturbed semilinear reaction-diffusion problem with discontinuous data. Appl. Math. Comput. 182, 244-257 (2006)

8. de Falco, C, O'Riordan, E: Interior layers in a reaction-diffusion equation with a discontinuous diffusion coefficient. Int. J. Numer. Anal. Model. 7, 444-461 (2010)

9. Rao, SCS, Chawla, S: Interior layers in coupled system of two singularly perturbed reaction-diffusion equations with discontinuous source term. Lect. Notes Comput. Sci. 8236, 445-453 (2013)

10. Brayanov, IA: Numerical solution of a two-dimensional singularly perturbed reaction-diffusion problem with discontinuous coefficients. Appl. Math. Comput. 182, 631-643 (2006)

11. Herceg, D: Uniform fourth order difference scheme for a singular perturbation problem. Numer. Math. 56, 675-693 (1990)

12. Linß, T: Robust convergence of a compact fourth-order finite difference scheme for reaction-diffusion problems. Numer. Math. 111, 239-249 (2008)

13. Sun, G, Stynes, M: An almost fourth order uniformly convergent difference scheme for a semilinear singularly perturbed reaction-diffusion problem. Numer. Math. 70, 487-500 (1995)

14. Vulanović, R: Fourth order algorithms for a semilinear singular perturbation problem. Numer. Algorithms 16, 117-128 (1997)

\section{Submit your manuscript to a SpringerOpen ${ }^{\circ}$ journal and benefit from:}

- Convenient online submission

- Rigorous peer review

- Open access: articles freely available online

- High visibility within the field

- Retaining the copyright to your article

Submit your next manuscript at $\boldsymbol{~ s p r i n g e r o p e n . c o m ~}$ 